\title{
OFDM óptimo para la comunicación bidireccional en las redes eléctricas inteligentes
}

\author{
OFDM optimal for bi-directional communication in smart grid \\ Washington Fernández R. ${ }^{1 *} \quad$ Arturo Rodríguez R. ${ }^{2}$ \\ Recibido 21 de junio de 2016, aceptado 15 de diciembre de 2016 \\ Received: June 21, 2016 Accepted: December 15, 2016
}

\section{RESUMEN}

En este artículo se diseña un sistema OFDM óptimo para la comunicación bidireccional en las redes eléctricas inteligentes. La transmisión se realiza simulando un canal con ruido clase A, el cual es característico de las líneas eléctricas de alto, medio y bajo voltaje.

El sistema OFDM es óptimo porque se emplean constelaciones bidimensionales rotadas: rectangular, circular y hexagonal de cuatro y dieciséis símbolos que tienen mejores desempeños comparados a sus contrapartes que son las constelaciones sin rotar.

El OFDM óptimo disminuye la probabilidad de error de bits como mínimo de $1 \times 10^{-2}$ cuando se comparan con OFDM-QAM, en un canal con ruido clase A.

Palabras clave: OFDM, constelaciones rotadas, redes eléctricas inteligentes, ruido clase A.

\section{ABSTRACT}

In this article is designed a system OFDM optimal for the bi-directional communication at the smart grid. The transmission is simulating a channel with noise class A, what is characteristic of the electrical lines of high, medium and low voltage.

The OFDM system is optimal because they employ two-dimensional constellations rotated: rectangular, circular and hexagonal of four and sixteen symbols that have the best performance compared to their counterparts who are the constellations without rotating.

The optimal OFDM decreases the likelihood of error of bits as minimum of $1 \times 10^{-2}$ when compared with OFDM-QAM, on a channel with noise class A.

Keywords: OFDM, constellations rotated, smart grid, class A noise.

\footnotetext{
1 Departamento de Ingeniería Eléctrica y Electrónica. Universidad del Bío-Bío. Concepción, Chile. E-mail:wfernand@ubiobio.cl

2 Departamento de Tecnología Industriales. Universidad de Santiago de Chile. Santiago, Chile. E-mail: Arturo.rodriguez@usach.cl

* Autor de correspondencia
} 


\section{INTRODUCCIÓN}

Redes eléctricas inteligentes es un término que se utiliza para referirse a la próxima generación de redes eléctricas, en la cual la distribución y manejo de la electricidad necesita de comunicación bidireccional y capacidad computacional para realizar supervisión, eficiencia, confiabilidad y seguridad [1, 2, 3, 4].

La red inteligente se espera que modernice la red eléctrica actual, lo cual implica supervisión, (se tendrá monitoreo en tiempo real del consumo eléctrico, estado de la red, calidad de la energía eléctrica suministrada por cada uno de los sistemas de generación eléctrica interconectados), protección y optimización automática de la operación de los sistemas interconectados a la red eléctrica (por ejemplo se colocan medidores inteligentes en la red de generación, transmisión y distribución, los cuales realizan cálculos de parámetros en tiempo real y toman auto decisión en caso de falla).

La Figura 1 muestra la concepción de una red eléctrica inteligente de acuerdo a [4]. Este modelo presenta tres tipos de consumidores: Red de Área de Casa (HAN: Home Area Network), Red de Área de Edificio (BAN: Building Area Network) y Red de Área de Industrial (IAN: Industrial Area Network). Dentro de estas áreas se tiene una Infrastructura Avanzada de Supervisión (AMI: Advanced Metering Infrastructure) es el despliegue para la supervisión del flujo de datos. En la Figura 2 se muestra por ejemplo el uso de AMI en las HAN's [4].

De acuerdo a lo expuestos en los párrafos anteriores, la red eléctrica requiere de una red bidireccional de comunicación de datos. Para realizar la comunicación se proponen varias infraestructuras, por ejemplo: que sean inalámbricas, sistema óptico o se emplee la misma línea eléctrica.

En este artículo se propone para la comunicación de datos en la red eléctrica inteligente un sistema de transmisión/rececpión utilizando un OFDM óptimo. El desempeño se determina para un canal de transmisión con la línea eléctrica de alto, medio y bajo voltaje, lo cual equivale a tener un ruido

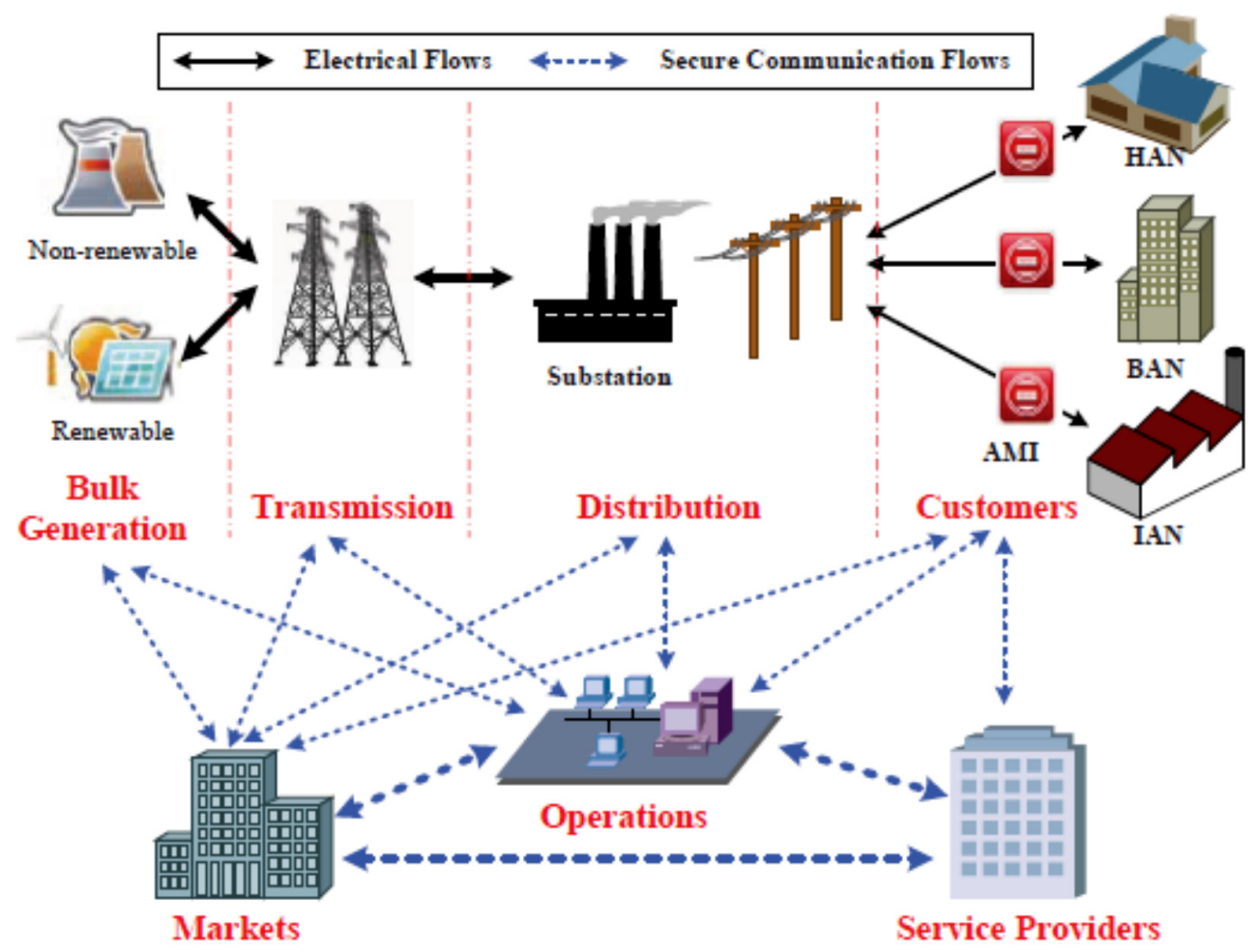

Figura 1. Concepción de una red eléctrica inteligente. 


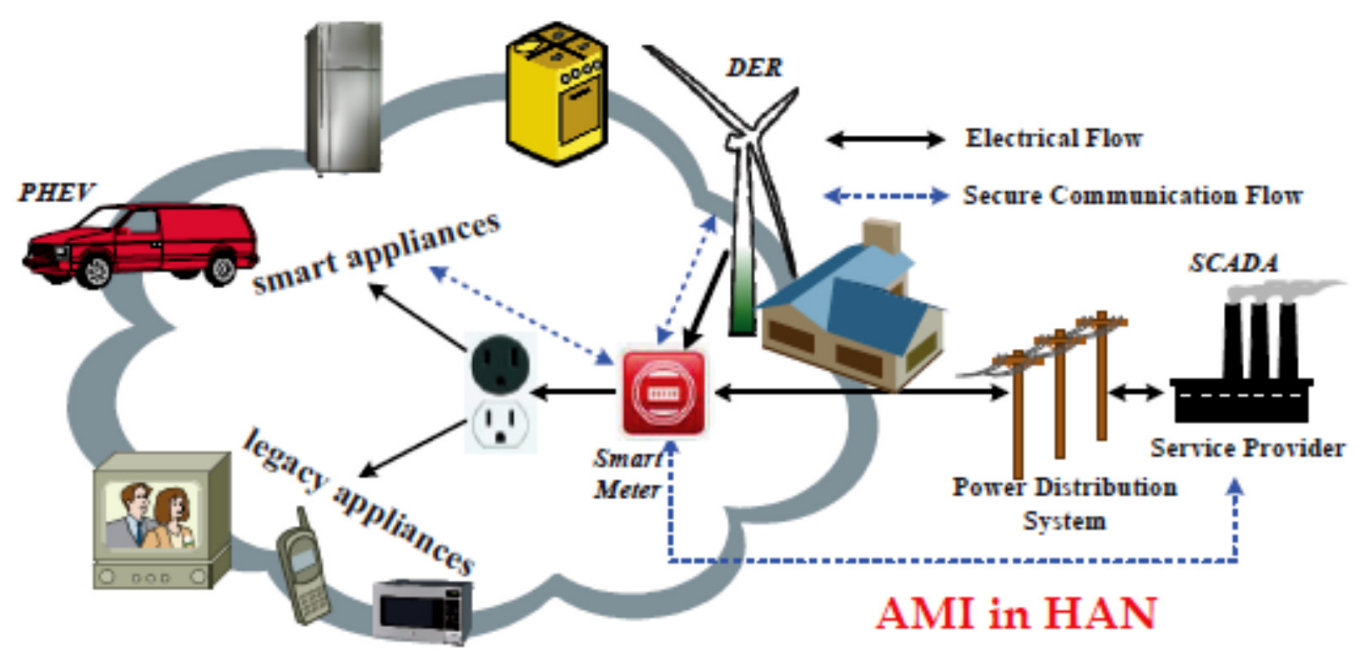

Figura 2. Uso de AMI en las HAN's.

clase A, este ruido está constituido por un ruido Gaussiano más un ruido impulsivo.

El sistema OFDM tiene la característica de mejorar la probabilidad de error de bits en canales muy adversos para la transmisión de datos.

Hace más de treinta años que se realiza investigación y desarrollo relacionado con multiplexación por división de frecuencias ortogonales (OFDM) [7].

El concepto de usar la transmisión de dato en paralelo por la multiplexación de división de frecuencia se publica en la mitad de los sesenta [8] y [9].

En OFDM cada portadora es ortogonal una de otra, lo cual no se mantiene en la técnicas con múltiples tonos discretos (DMT) [10].

OFDM se basa en el uso de M-QAM, M-PSK, [11]-[13]. En [14] se introduce el concepto de prefijo cíclico.

Con relación a las constelaciones bidimensionales se tiene el trabajo de [15] que estudia las constelaciones que minimiza la probabilidad de error en un canal con ruido clase A. En [16] se dan las técnicas para generar y optimizar un conjunto de señales hexagonales. En [17] se presenta el ángulo óptimo para modulaciones 8-PSK y 16-QAM, para lograrlo minimiza una función de costo. En [18] se da una expresión analítica para la rotación óptima de ángulos con el criterio de la distancia mínima Euclidiana para cualquier constelación M-PSK.

Middleton clasifica el ruido en tres clases:

Clase A: Este ruido es típicamente de espectro de banda angosta. Es decir, más angosto que el ancho de banda del receptor.

Clase B: Aquí el ancho de banda del ruido que llega al receptor tiene un mayor ancho de banda que el receptor.

Clase C: Es la suma del ruido clase A y B.

Un modelo de ruido se sugiere en [19], en el cual se tiene:

$$
P_{Z}(z)_{P+G} \approx e^{-A} \sum_{m=0}^{\infty} \frac{A^{m}}{m ! \sqrt{2 \pi \sigma_{m}^{2}}} e^{-\frac{z^{2}}{2 \sigma_{m}^{2}}}
$$

Donde:

$$
\sigma_{m}^{2}=\frac{c_{m}^{2}}{\Omega_{2 A}(1+\Gamma)}=\frac{\frac{m}{A}+\Gamma}{1+\Gamma}
$$

Para el ruido clase A:

$$
\Gamma=\frac{\sigma_{G}^{2}}{\sigma_{I}^{2}}
$$


Donde:

$\sigma_{G}^{2}:$ Varianza Gaussiana.

$\sigma_{I}^{2}$ : Varianza Impulsiva.

Este modelo de ruido corresponde a un proceso aleatorio, discreto, independiente y distribuido idénticamente (iid), el cual tiene características muy diferentes desde la clásica interferencias Gaussiana. Las redes eléctricas de alto, medio y bajo voltaje como las HAN, BAN y IAN presentan un ruido clase A.

La organización del trabajo es la siguiente: en la Sección II se analiza el sistema de transmisión/ recepción propuesto. En la Sección III se presentan los resultados encontrados en forma de gráficos y se realiza una discusión de los resultados. Finalmente, se pueden encontrar las conclusiones en la Sección IV.

\section{ANÁLISIS DEL SISTEMA PROPUESTO DE TRANSMISIÓN/RECEPCIÓN}

Un sistema de comunicación que se basa en OFDM, transmite múltiples datos simultáneamente y usa sub portadoras ortogonales para ello. Los símbolos de dato $d_{n, k}$ primero se agrupan en un bloque de tamaño $N$ y entonces se modulan con formas de ondas exponenciales complejas $f_{k}(t)$, las cuales se implementan utilizando la transformada rápida inversa de Fourier (IFFT).

Matemáticamente se expresa como:

$$
x(t)=\sum_{n=-\infty}^{\infty}\left[\sum_{k=0}^{N-1} d_{n, k} \phi_{k}\left(t-n T_{d}\right)\right]
$$

Donde:

$$
\phi_{k}(t)=\left\{\begin{array}{l}
e^{j 2 \pi f_{k} t} \quad t \in\left[0, T_{d}\right] \\
0 \quad \text { De otra manera }
\end{array}\right.
$$

Y:

$$
f_{k}=f_{o}+\frac{k}{T_{d}} \quad k=0, \ldots \ldots, N-1
$$

$d_{n, d}$ : Símbolo que se transmite durante el intervalo $n$-ésimo de tiempo usando la $k$-ésima sub portadora.
$T_{d}$ : Duración del símbolo.

$N$ : Número de sub portadoras OFDM.

$f_{k}$ : Frecuencia de la $k$-ésima sub portadora.

$f_{0}$ : Frecuencia más baja.

El proceso de demodulación se basa en la ortogonalidad de las sub portadoras $f_{k}(t)$, se tiene:

$$
\int_{\Re} \phi_{k}(t) \phi_{l}(t) d t=T_{d} \delta(k-l)=\left\{\begin{array}{ll}
T_{o} & k=1 \\
0 & \text { De otra manera }
\end{array}\right. \text { (7) }
$$

Por lo tanto, el demodulador se implementa explotando la ortogonalidad de las sub portadoras digitalmente se implementa con la transformada rápida de Fourier (FFT).

$$
d_{n, k}=\frac{1}{T_{d}} \int_{n T_{d}}^{(n+1) T_{d}} x(t) \phi_{k}(t) d t
$$

A continuación se analizan las constelaciones bidimensionales rotadas.

Una constelación con dimensión $N$ es un conjunto finito de $\mathrm{N}$-uplas o puntos en el espacio dimensional $N$. El tamaño de la constelación $\mid C l$ es el número de puntos. En una constelación bidimensional se tiene $|C|=2^{b}$ puntos, con $b$ número entero.

Sea una constelación en el plano complejo, es decir, de la forma: $q_{i}= \pm x_{i} \pm y_{i}$, como se muestra en la Figura 3.

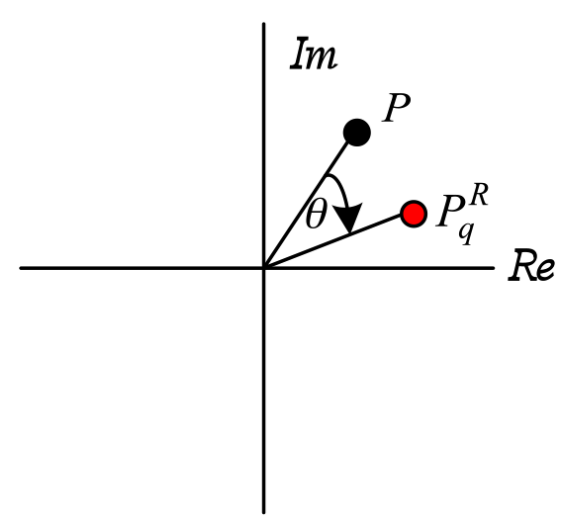

Figura 3. Puntos en el plano complejo.

De acuerdo a la Figura 3, los puntos $P$ y $P_{q}^{R}$ se relacionan con la siguiente transformación: 


$$
\left[P_{q}^{\mathrm{Re}} P_{q}^{\mathrm{Im}}\right]=\left[P_{\mathrm{Re}} P_{\mathrm{Im}}\right]\left[\begin{array}{lr}
\cos \theta-\operatorname{sen} \theta \\
\operatorname{sen} \theta & \cos \theta
\end{array}\right]
$$

Si el ángulo se mide en el sentido horario los puntos se escriben como:

$$
\begin{aligned}
& x_{i}=a_{i} \cos \theta-b_{i} \operatorname{sen} \theta \\
& y_{i}=a_{i} \operatorname{sen} \theta+b_{i} \cos \theta
\end{aligned}
$$

Donde:

$a_{i}$ : Punto de la constelación sin rotar, eje real.

$b_{i}$ : Punto de la constelación sin rotar, eje imaginario.

Para determinar el símbolo que se transmite, el receptor utiliza la métrica:

$$
d_{i}=\left|r_{i}-x_{i}\right|^{2}+\left|r_{2}-y_{i}\right|^{2}
$$

Donde:

$r_{1}$ : Parte real de símbolo que se recibe en el receptor.

$\mathrm{r}_{2}$ : Parte imaginaría del símbolo que se recibe en el receptor.

$x_{i}$ : Símbolo de la constelación parte real.

$y_{i}$ : Símbolo de la constelación parte imaginaría.

El detector escoge a favor del símbolo $s_{i}=\left(w_{i}, y_{i}\right)$ que minimiza a (12).

De [15], se tiene la métrica:

$$
P_{e}\left(s_{i} \rightarrow \hat{s}_{i}\right) \leq \frac{1}{2}\left(\frac{1}{1+\left|x_{i}-\hat{x}_{i}\right|^{2}}\right)\left(\frac{1}{1+\left|y_{i}-\hat{y}_{i}\right|^{2}}\right)
$$

Donde:

$P_{e}: \quad$ Probabilidad de error de símbolo.

$s_{i}$ : $\quad$ Símbolo de la constelación sin rotación de ángulo.

$\hat{S}_{i}$ : Símbolo de la constelación con rotación de ángulo.

$x_{i}, y_{i}$ : Coordenadas del símbolo sin rotación de ángulo.

$\hat{x}_{i}, \hat{y}_{i}$ : Coordenadas del símbolo con rotación de ángulos.
Si se utilizan (10), (11) y se reemplaza en (13), se logra:

$$
P_{e}\left(s_{i} \rightarrow \hat{s}_{i}\right) \geq G_{1} G_{2}
$$

Donde:

$$
G_{1}=\frac{1}{1+\left|\left(a_{i}-\hat{a}_{i}\right) \cos \theta+\left(b_{i}-\hat{b}_{i}\right) \operatorname{sen} \theta\right|^{2}}
$$

$$
G_{2}=\frac{1}{1+\left|\left(a_{i}-\hat{a}_{i}\right) \operatorname{sen} \theta+\left(b_{i}-\hat{b}_{i}\right) \cos \theta\right|^{2}}
$$

Cuando no hay rotación:

$$
\begin{gathered}
\theta=0 \\
x_{i}=a_{i}
\end{gathered}
$$

$$
y_{i}=b_{i}
$$

En este caso $G_{1}$ y $G_{2}$ son:

$$
\begin{aligned}
& G_{1}=\frac{1}{1+\left|\left(a_{i}-\hat{a}_{i}\right)\right|^{2}} \\
& G_{2}=\frac{1}{1+\left|\left(b_{i}-\hat{b}_{i}\right)\right|^{2}}
\end{aligned}
$$

Si se compara (15) con (10) y (16) con (21), se observa que la probabilidad de error depende del ángulo de rotación $q$, la tarea es encontrar el valor de $q$ que minimice la probabilidad de error.

Para minimizar la probabilidad de error es equivalente a minimizar la función de costo dada por (22):

$$
J=\sum_{\hat{s}_{i} \neq s_{i}} P_{e}\left(s_{i} \rightarrow \hat{s}_{i}\right)
$$

La minimización de la función de costo $J$ se realiza numéricamente usando el algoritmo de gradiente que se indica en (23): 


$$
\theta(k+1)=\theta(k)+u \frac{d J}{d \theta}
$$

Donde:

$u$ : Constante de valor pequeño.

$q$ : Valor inicial.

Evaluando (22) y (23) en forma exhaustiva se obtienen los ángulos de rotaciones óptimos para cada una de las constelaciones rectangular, hexagonal y circular para cuatro y dieciséis símbolos, los cuales se indican en la Tabla 1.

Tabla 1. Ángulos de rotaciones óptimos para las constelaciones rectangular, circular y hexagonal para ruido clase $\mathrm{A}$.

\begin{tabular}{|r|c|c|c|}
\hline $\mathbf{N}$ & Rectangular & Circular & hexagonal \\
\hline 4 & $10^{\circ}$ & $38^{\circ}$ & $25^{\circ}$ \\
\hline 16 & $8^{\circ}$ & $20^{\circ}$ & $18^{\circ}$ \\
\hline
\end{tabular}

La Figura 4 muestra el sistema de transmisión que se propone para disminuir la probabilidad de error de bits, de acuerdo a la Figura 4, se tienen los datos a transmitir $d(t)$, el mapeo de constelaciones rotadas que convierten los datos binarios a símbolos $m(t)$ de la constelación rectangular, circular, hexagonal de cuatro y dieciséis símbolos que se utilice, luego se realiza una conversión de los símbolos serial a paralelo $p(t)$ a continuación para transmitir $N$ sub portadoras se aplica la transformada rápida inversa de Fourier ift $(t)$, posteriormente se le agrega un prefijo cíclico que consiste en los últimos 6 símbolos que se transmiten $p(t)$.

La Figura 5 muestra el receptor donde se sacan los prefijos cíclicos $s p(t)$, se aplica la transformada rápida de Fourier $f f t(t)$, el estimador de símbolo de máxima probabilidad, estima que símbolo se transmite $\hat{m}(t)$, a continuación se realiza la conversión de los símbolos de paralelo a serial $\hat{s}(t)$, para finalmente el desmapeo de constelaciones rotadas lleva los símbolos a datos binarios de acuerdo a la constelación rectangular, circular, hexagonal de cuatro y dieciséis símbolos que se transmiten.

La Figura 6 muestra el sistema de transmisión/ recepción que consiste del transmisor, el canal de transmisión que le suma ruido blanco, aditivo, Gaussiano más ruido impulsivo y el receptor.

Para determinar el desempeño del OFDM óptimo, en primer lugar se envían los datos digitales mediante el transmisor, pasan por el canal con ruido clase A y se reciben en el receptor, donde se estiman los datos que se transmiten. Se comparan los bits transmitidos con los recibidos y se determinan cuantos bits se recibieron erróneos de esta forma se obtiene el BER, esto se hace para cada valor de SNR, partiendo de cero dB y con un incremento de

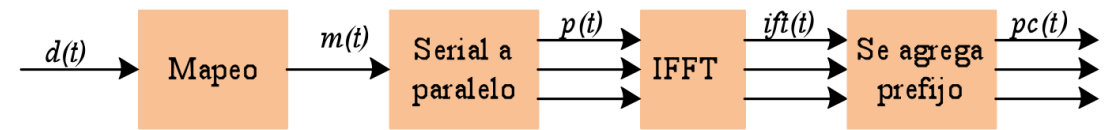

Figura 4. Esquema del transmisor de OFDM óptimo.

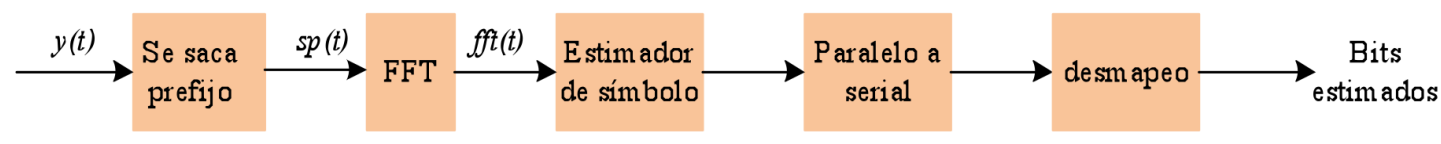

Figura 5. Esquema del receptor de OFDM óptimo.

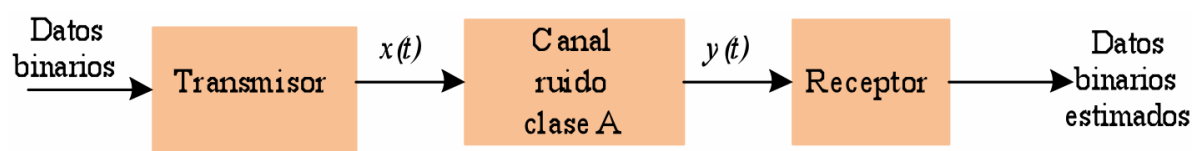

Figura 6. Esquema para determinar el desempeño del OFDM óptimo. 
dos decibeles, con los valores tabulados se hacen las graficas BER versus SNR.

\section{RESULTADOS Y DISCUSIÓN}

\section{Resultados}

Los parámetros del ruido para el canal con línea eléctrica se consideran: $A=0.01, \Gamma=0.001$ y $m=3$, con estos valores el ruido es altamente impulsivo.
La Figura 7 muestra las características del ruido que se simula y la Figura 8 el ruido que se mide en una línea eléctrica.

En las Figuras 9 y 10 se muestran las comparaciones del desempeño 4-QAM-OFDM con OFDM óptimo (constelación rotadas de rectangular, circular y hexagonal de cuatro símbolos) con 64 y 1024 sub portadoras respectivamente.

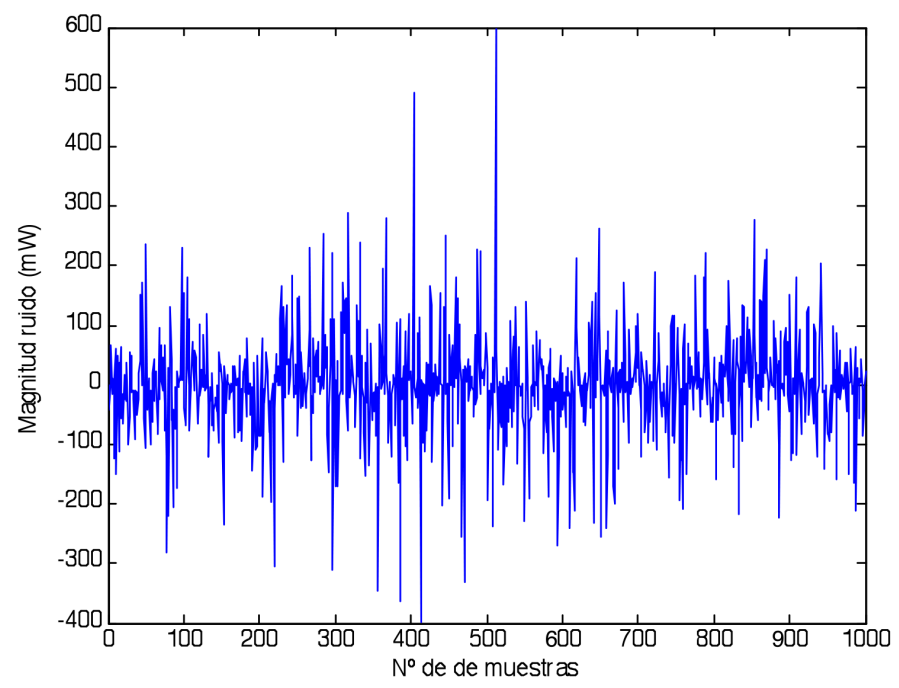

Figura 7. Característica del ruido presente en la línea eléctrica.

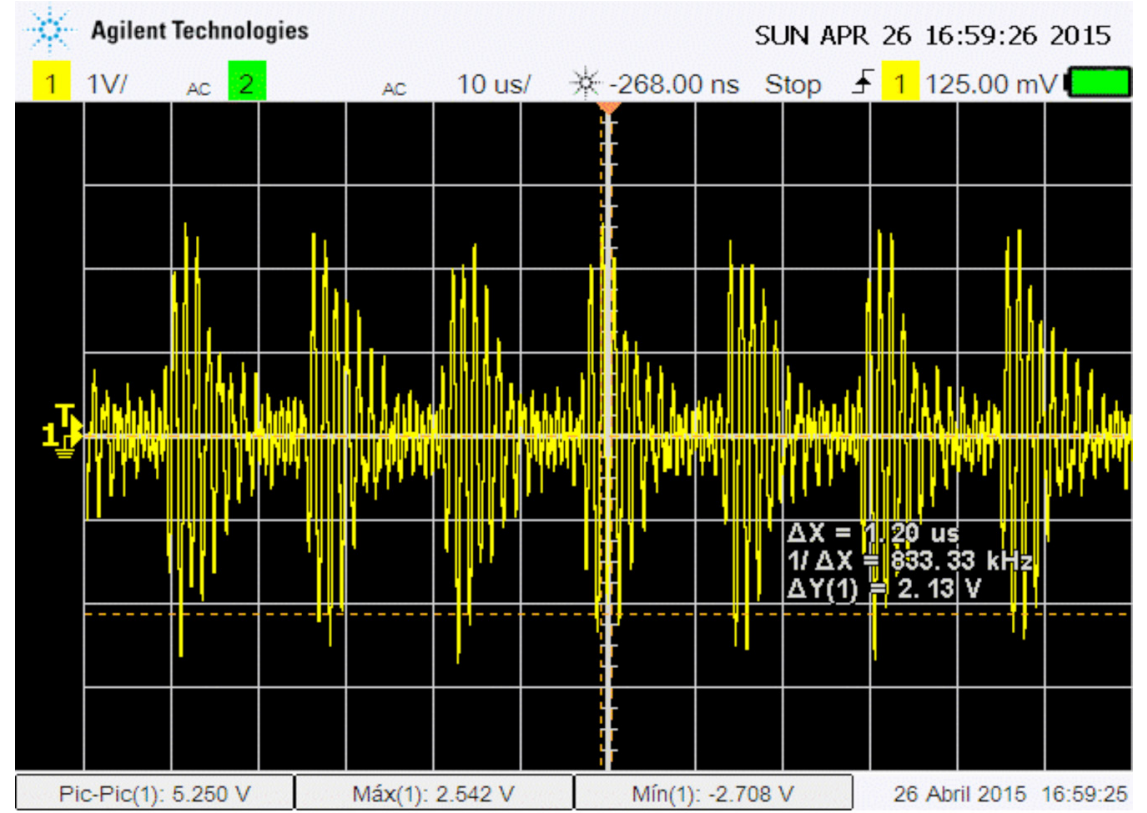

Figura 8. Característica del ruido medido en la línea eléctrica de bajo voltaje. 

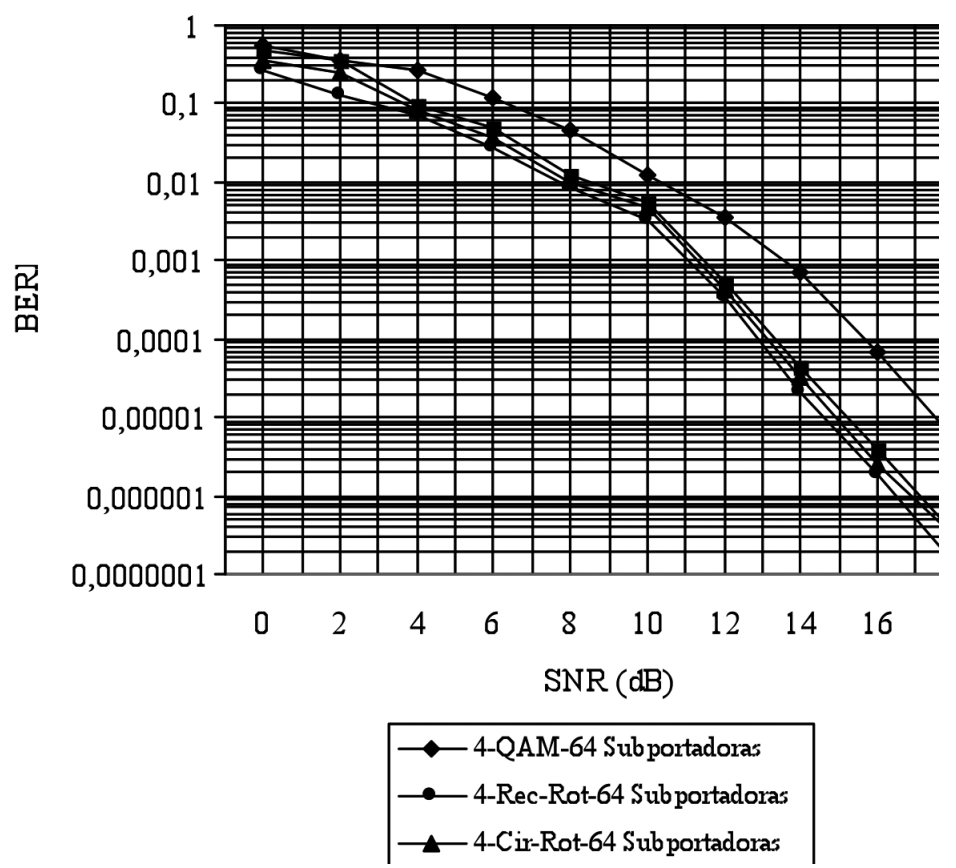

Figura 9. Resultados de SER versus SNR para la constelación rotada rectangular, circular y hexagonal de cuatro símbolos y OFDM 4-QAM con sesenta y cuatro sub portadoras.
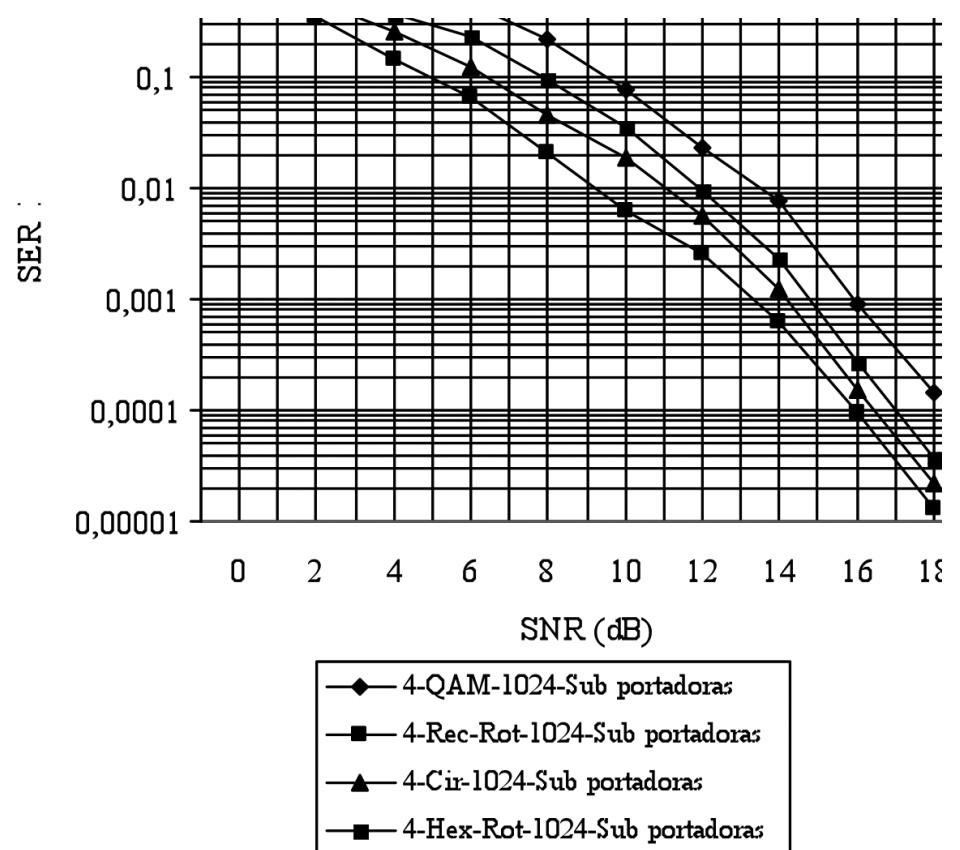

Figura 10. Resultados de SER versus SNR para la constelación rotada rectangular, circular y hexagonal de cuatro símbolos y OFDM 4-QAM con mil veinticuatro sub portadoras. 
En las Figuras 11, 12 y 13 se muestran las comparaciones del desempeño 16-QAM-OFDM con OFDM óptimo (constelación rotadas de rectangular, circular y hexagonal de dieciséis símbolos) con 64, 256 y 1024 sub portadoras respectivamente.

\section{Discusión de resultados}

La Figura 7 indica que con los valores de los parámetros que se dan el ruido es altamente impulsivo, el cual es la característica del canal de transmisión con líneas eléctricas, lo que se demuestra con el ruido que se mide (Figura 8 ).

De las Figuras 9 y 10, de la 11 a la 13 se infiere que la rotación de las constelaciones: rectangular, circular y hexagonal mejoran notablemente el desempeño de OFDM en un canal con ruido Gaussiano y ruido impulsivo (ruido clase $\mathrm{A}$ ) disminuyendo la probabilidad de error de símbolo, la causa de esto es porque el ángulo óptimo de rotación permite que la ubicación en el plano dimensional de los símbolos de las constelaciones aumente la región de decisión entre ellas, lo que provoca a su vez que el detector de máxima probabilidad aumenta la probabilidad de estimar el símbolo en forma correcta, lo que incide en mejorar el desempeño de la modulación OFDM, esto se ve mejor reflejado con las constelaciones rectangular y hexagonal (Figuras 7 al 11).

Si se compara el desempeño del OFDM óptimo con otros sistemas por ejemplo con [15], se observa que se logra una mejora en el BER para el mismo SNR de un orden de magnitud como mínimo, esto se debe al sistema OFDM que mejora el desempeño en un canal con ruido impulsivo, lo mismo sucede si se compara con [13] y [17], esto se debe a que la rotación de las constelaciones rectangular, circular y hexagonal, disminuyen la probabilidad de error de bits.

\section{CONCLUSIONES}

De acuerdo a los resultados que se obtienen el OFDM óptimo que se diseña y construye en este trabajo disminuye en forma muy significativa la probabilidad de error de símbolo en comparación a su contraparte QAM y es un buen candidato para

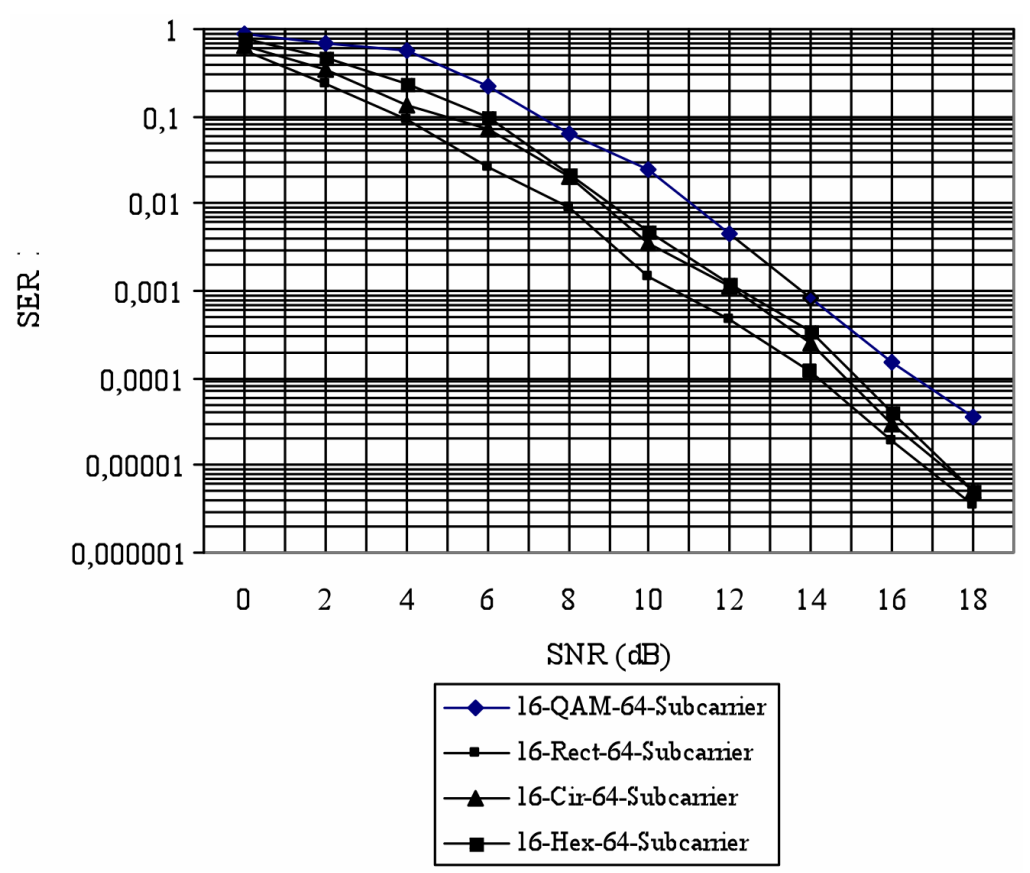

Figura 11. Resultados de SER versus SNR para la constelación rotadas rectangular, circular y hexagonal de dieciséis símbolos y OFDM 16-QAM con sesenta sub portadoras. 


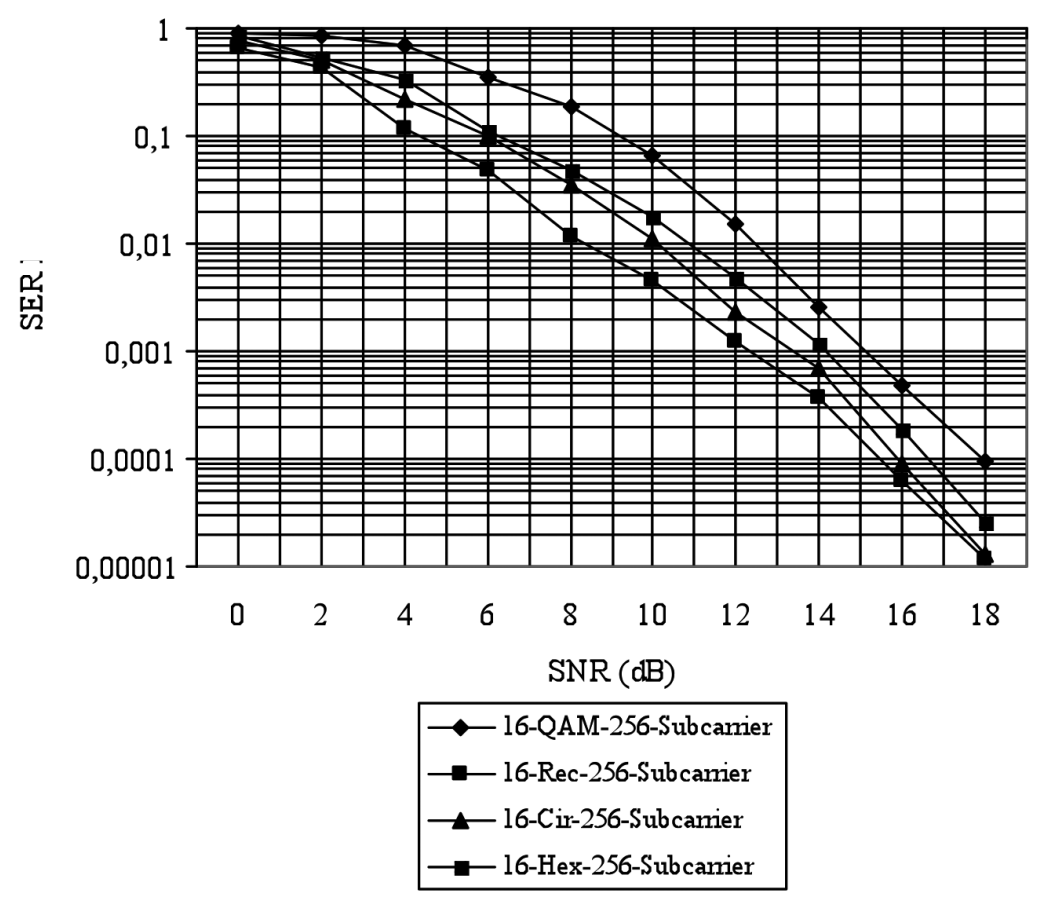

Figura 12. Resultados de SER versus SNR para la constelación rotada rectangular, circular y hexagonal de dieciséis símbolos y OFDM 16-QAM con doscientas cincuenta y seis sub portadoras.

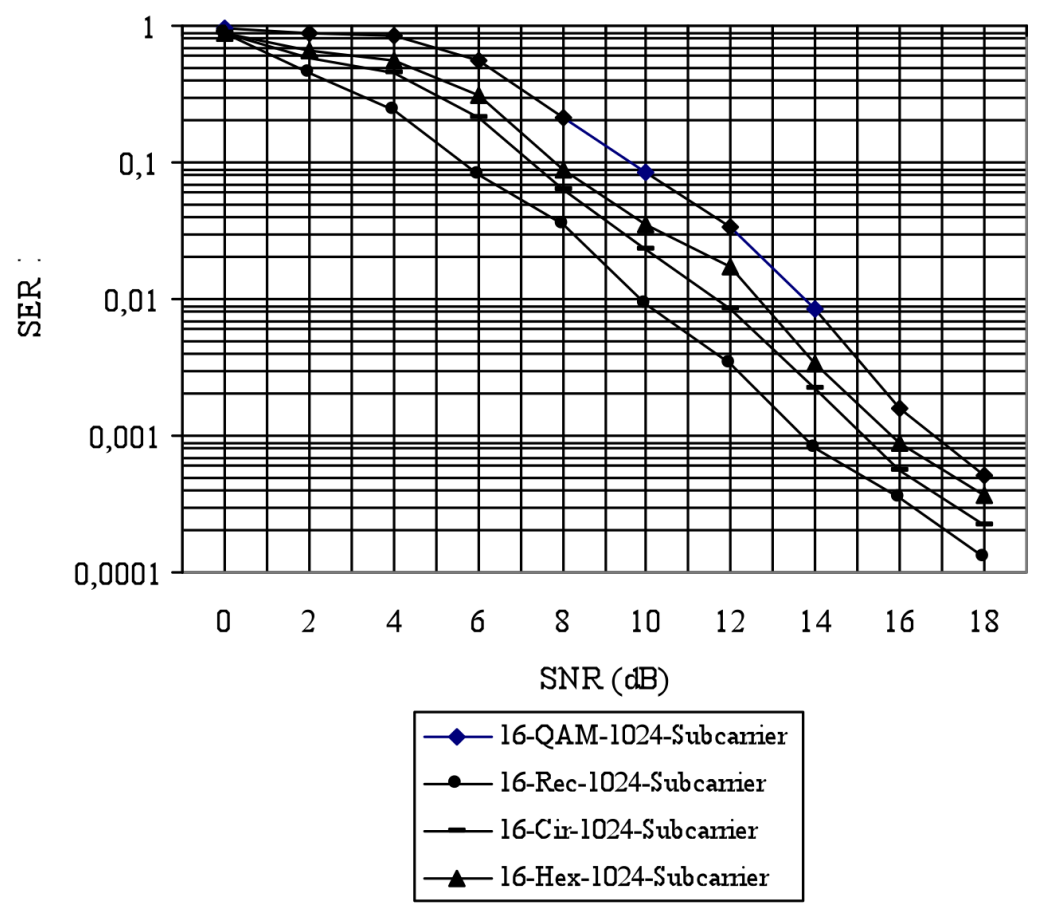

Figura 13. Resultados de SER versus SNR para la constelación rotada rectangular, circular y hexagonal de dieciséis símbolos y OFDM 16-QAM con mil veinticuatro sub portadoras. 
que se implemente en la transmisión de dato para las redes eléctricas inteligentes (HAN, BAN e IAN).

\section{AGRADECIMIENTOS}

Los autores agradecen a los evaluadores por sus comentarios y sugerencias, las cuales permiten enriquecer aún más este artículo.

Se agradece a la Universidad del Bío-Bío mediante el proyecto de investigación interno $\mathrm{N}^{\circ} 170810$ 3/R y a FAPEI, como también al Proyecto Basal MECESUP-USACH USA1555 por apoyar la realización de este artículo.

\section{REFERENCIAS}

[1] Departament of energy, The smart grid and introduction, 2010, disponible en: www. energy.gov/SmartGridIntroduction.html.

[2] Y. Kim, H. Thottan and W. Lee, A Secure Descentralized Data Centric Information infrastructure for smart grid, IEEE Communications Magazine, pp. 58-65, June 2010.

[3] H. Gharavi, R. Ghafurian, Smart Grid the Electric System of Future, Proccending of IEEE, pp. 917-921, August 2011.

[4] K. Chen, P. Yeh, H. Hsi and S. Chang, Communication infrastructure of smart grid, 4th International Symposium Control and Signal Processing (ISCCP), pp. 1-5, 2010.

[6] Z. Fan, P, Smart grid communication: overview of research challenge solutions, and standardization activities, IEEE Communications Survey \& Tutorial, Vol. 25, $\mathrm{N}^{\circ} 1$, pp. 21-33, First Quarter 2012.

[7] J. Bingham, Multicarrier Modulation for Data Transmission: An Idea Whose Time Has Come, IEEE Communications Magazine, pp. 5-16, May 1990.

[8] R. Chang, Synthesis of Band-limited Orthogonal Signals for Multichannel data Transmission, Bell Syt. Tech. J., Vol. 45, pp. 1775-1796, December 1966.

[9] B. Saltzberg, Performance of an Efficient Parallel Data Transmission System, IEEE Transactions Communications Technology, Vol. 15, December 1967.
[10] W. Zou, Y. Wu, COFDM: An Overview, IEEE Transactions on Broadcasting, Vol. 41, $\mathrm{N}^{\circ} 1$, pp. 1-8, March 1995.

[11] L. Cimini, and G. Li, Orthogonal Frequency Division Multiplexing For Wireless Communications, Tutorial, IEEE Vehicular Technology Conf., (VTC'00), 2000.

[12] J. Patel, U. Dalal, A Comparative Performance Analysis of OFDM Using MATLAB Simulation with M-PSK and M_QAM Mapping, International Conference on Computational Intelligence and Multimedia Applications, pp. 406-410, 2007.

[13] Z. Wang, OFDM or Single Carrier Blosk Transmission, IEEE Transactions on Communication, Vol. 52, No 3 , pp. 480494, March 2004.

[14] A. Pelled and R. Ruiz, Frequency Domain Data Transmission Using Reduced Computational Complexity Algorithms, Proceedings IEEE Int. Conf. On Acoustics, Speech and Signal Processing (ICAAP' 80) pp. 964-967, Denver Co, 1980.

[15] W. Fernández, Estudio del Desempeño de las Constelaciones Hexagonal, Circular y Rectangular con Entrelazador Dorado para Líneas Eléctricas Inteligentes, IEEE Latín América Transactions, Vol. 12, No 8 , pp. 1402-1408, December 2014.

[16] K. Simon and J. Smith, Hexagonal, Multiple Phase and Amplitude Shift Keyed Signal Set, IEEE Transactions on Communications, Vol. 2, No 10 , pp. 1108 115, October 1973.

[17] L. Leszek and M. Bacic, Constellations Design for Multiple Transmissions Maximizing the Minimum Squared Euclidean Distance, IEEE Wireless Communications and Networking Conference, Vol. 2, pp. 1066-1071, March 2005.

[18] G. Forney Jr, L.F. Wei, Multidimensional Constellations Part I: Introduction, Figures of Merit and Generalized Cross, IEEE Journal on Selected Areas in Communications, Vol. 7 , $\mathrm{N}^{\circ} 6$, pp. 877-892, August 1989.

[19] L. Di Bert, D. Schwingshack, and A. Tonello, On Noise Modeling for Power Line Communications, IEEE International Symposium on Power Line Communications and Applications, pp. 283-287, 2011. 